PAPER • OPEN ACCESS

\title{
Symmetries and criticality of generalised van der Waals models
}

To cite this article: Francesco Giglio et al 2021 J. Phys. A: Math. Theor. 54405701

View the article online for updates and enhancements.

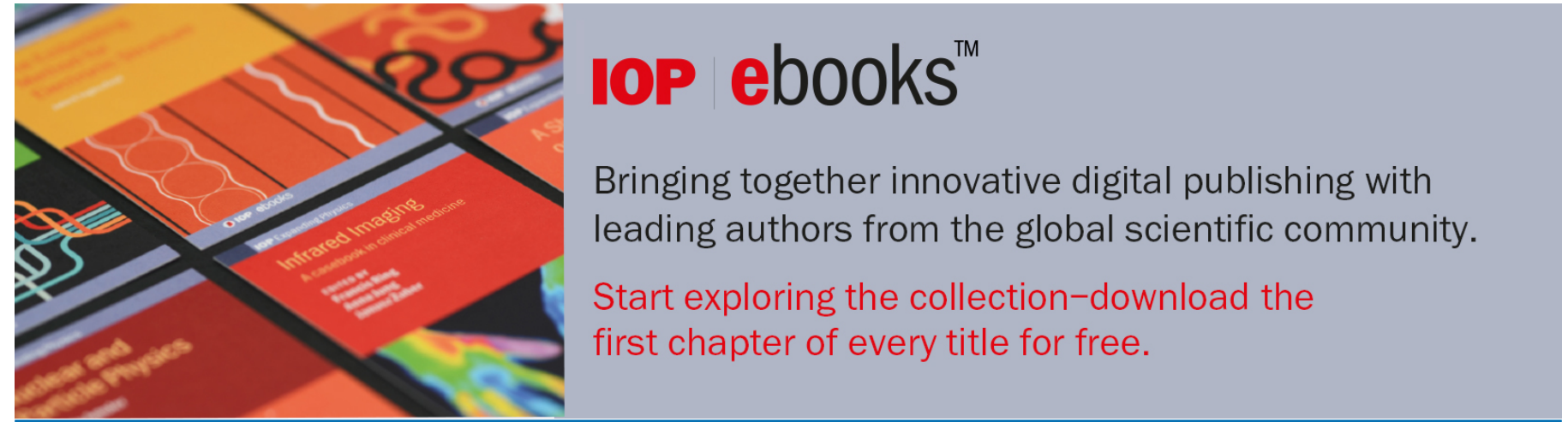

This content was downloaded from IP address 130.209 .116 .71 on 13/09/2021 at 12:47 


\title{
Symmetries and criticality of generalised van der Waals models
}

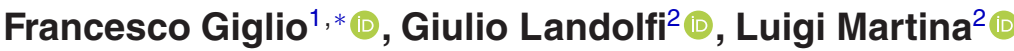 \\ and Antonio Moro ${ }^{3}$
}

${ }^{1}$ School of Mathematics and Statistics, University of Glasgow, Glasgow, United Kingdom

2 Dipartimento di Matematica e Fisica 'Ennio De Giorgi’ Università del Salento, I.N.F.N. Sezione di Lecce, via Arnesano I-73100 Lecce, Italy

${ }^{3}$ Department of Mathematics, Physics and Electrical Engineering, Northumbria

University Newcastle, United Kingdom

E-mail: francesco.giglio@glasgow.ac.uk,giulio.landolfi@le.infn.it, luigi.martina@le.infn.it and antonio.moro@northumbria.ac.uk

Received 28 May 2021, revised 7 August 2021

Accepted for publication 23 August 2021

Published 9 September 2021

\begin{abstract}
We consider a family of thermodynamic models such that the energy density can be expressed as an asymptotic expansion in the scale formal parameter and whose terms are suitable functions of the volume density. We examine the possibility to construct solutions for the Maxwell thermodynamic relations relying on their symmetry properties and deduce the critical properties implied in terms of the dynamics of coexistence curves in the space of thermodynamic variables.

Keywords: symmetries of differential equations, critical points and phase transitions, van der Waals type systems, shock waves
\end{abstract}

(Some figures may appear in colour only in the online journal)

\section{Introduction}

The study of equilibrium and critical phenomena in fluids is a widely addressed research subject. Fluids display indeed a rich phenomenology and a number of equations of state have been derived to effectively encode complex microscopic processes occurring as thermodynamical

*Author to whom any correspondence should be addressed.

cc) Original content from this work may be used under the terms of the Creative Commons Attribution 4.0 licence. Any further distribution of this work must maintain attribution to the author(s) and the title of the work, journal citation and DOI. 
conditions vary. The celebrated van der Waals equation of state

$$
\left(P+\frac{a}{v^{2}}\right)(v-b)=k_{\mathrm{B}} T
$$

(where $v$ denotes the volume density, $P$ the pressure, $T$ the temperature and $k_{\mathrm{B}}$ the Boltzmann constant) has played a pivotal role in this context, and constitutes a paradigm on which the vast majority of phenomenological models have been based and findings of theoretically based approaches have been tested, see e.g. [1-5]. Experimental investigations indicate in fact that a single equation of state cannot account of thermodynamic properties and phase transitions for all fluid systems, both pure and mixtures, under any conditions, and many empirical modifications of the van der Waals equations of state (1) have been devised for applications mainly in the context of chemical-engineering experiments [6-11]. A number of studies have also been carried out with the aim to link phenomenological models to specific assumptions on the form of the molecular interaction potential, see e.g. [12-20].

From the point of view of statistical mechanics phase transitions occur in the thermodynamic regime, the limit where both volume and number of particles diverge in such a way that the density stays finite. In this regime even 'well behaved' analytical potentials may lead to the occurrence of singularities in the dependence of physical observables as functions of thermodynamic variables. The classical macroscopic approach, based on the principles of thermodynamics, permits a direct description of macroscopic extensive and intensive thermodynamic variables via a set of differential equations known as Maxwell's relations. Maxwell's relations are equivalent to the existence of a free energy potential and specific assumptions on the functional form of the state functions enable one to derive the equations of state as solutions of these relations under suitable initial/boundary conditions [21, 22]. In this framework, fluid phase transitions, for example, can be interpreted as compressive shock waves in the profile of the state functions as they evolve in the space of thermodynamic variables [21, 23]. Shocks therefore arising as discontinuities in the profile of the state function correspond to the coexistence lines of two phases, see e.g. [21, 22, 24].

The advantage of the deployment of effective macroscopic models based of the analysis of Maxwell's relations, as outlined above, allows for an analytical description of a general class of phenomena by-passing the complications related to the development and implementation of extensive numerical simulations of underlying statistical mechanical models. A compelling example of the effectiveness of this approach is provided in [25] where a four parameter family of generalised van der Waals models results from a suitable class of internal energy virial expansions. In particular, it is found that the volume density $v(x, t ; \eta)$ satisfies the following nonlinear partial differential equation

$$
\frac{\partial v}{\partial t}+\frac{\partial}{\partial x}\left\{\frac{1}{c_{1} v-c_{3} k_{\mathrm{B}}}\left[c_{2} v^{2}+c_{4} k_{\mathrm{B}}^{2}-\eta k_{\mathrm{B}}\left(c_{1} \frac{\partial v}{\partial t}+c_{2} \frac{\partial v}{\partial x}\right)\right]\right\}=0
$$

with $x=P / T$ and $t=1 / T$, while the $c_{j}$ 's are structural constants entering the energy expansion. The quantity $\eta=1 / N$, where $N$ is the number of molecules, plays the role of the small parameter in the expansion of the internal energy. For the particular choice $c_{1}=0$ the model equation (2) (a Maxwell thermodynamic relation, see section 2) is identified with the well known Bateman-Burgers equation which describes the propagation of nonlinear waves in regime of small viscosity [26]. One thus expects that as $\eta \rightarrow 0$ a generic (physical) volume density solution changes into a shock wave at finite $t$, corresponding to the occurrence of the gas-liquid phase transition $[21,22]$. The occurrence of critical points and their configurations depend on the values of the structural constants $c_{j}$ and specific numerical values can be chosen 
to reproduce isothermal curves of various models, e.g. van der Waals, Soave-Redlich-Kwong and Peng-Robinson phenomenological models [25].

In this paper, we propose a systematic study of the model equation (2), its solutions obtained via the application of the Lie symmetry approach (see e.g. [27-29]) and their thermodynamic interpretation. The paper is organised as follows: in section 2 we introduce the model underpinned by the equation (2) and highlight its main features of interest in the realm of gas thermodynamics. In section 3 we present the results entailed by the Lie-symmetry analysis of the equation. In section 4 we discuss the application of results to equations of state and critical points for fluid systems. Last section is devoted to closing remarks.

\section{Generalised van der Waals model}

The nonlinear differential equation (2) for the volume density $v=v(x, t ; \eta)$ originates within the framework of laws of thermodynamics by considering the thermodynamic energy balance equation in the form $\mathrm{d} \psi=\varepsilon \mathrm{d} t+v \mathrm{~d} x$, where $\psi=t \mu$, where $\mu$ is the chemical potential and $\varepsilon=E / N$ is the internal energy per particle, being $N$ the total number of particles in the system. In these variables, the Maxwell relation, locally equivalent to the existence of the thermodynamic potential $\psi$, reads as

$$
\frac{\partial v}{\partial t}=\frac{\partial \varepsilon}{\partial x}
$$

Following a suggestion in [21], one can consider Maxwell thermodynamical equations within a nonlocal perturbative scheme where the expansion parameter is the inverse of the number of molecules $N$. More precisely, it is assumed that the internal energy density $\varepsilon$ admits the asymptotic expansion of the form

$$
\varepsilon=\varepsilon_{0}(v)+\eta \varepsilon_{1}(v) \frac{\partial v}{\partial x}+\eta \varepsilon_{2}(v) \frac{\partial v}{\partial t}+O\left(\eta^{2}\right)+h(t)
$$

where $\eta=1 / N$ and the function $h(t)$ is an arbitrary function of its argument. The derivatives of the volume density in the internal energy density expansion are introduced based on the observation that a number of perturbative approaches applied to the 12-6 Lennard-Jones potential [3], as well as more general type of potentials, lead to equations of state which depend on derivatives of the volume densities such as compressibility and thermal expansion coefficient.

In [25], a class of internal energy virial expansions (4) has been obtained by requiring that the Maxwell thermodynamical relation (3) is linearisable via the Cole-Hopf transformation

$$
v(x, t ; \eta)=-\eta k_{\mathrm{B}} \frac{\partial \log \varphi(x, t ; \eta)}{\partial x}
$$

This mathematical constraint provides, based on the form of the expansion (4), a natural C-integrable generalisation [30] of the van der Waals model [25] which, therefore, admits infinitely many conserved quantities. This constraint restricts the energy terms in equation (4) to the following form

$$
\varepsilon_{0}=-\frac{c_{2} v^{2}+c_{4} k_{\mathrm{B}}^{2}}{c_{1} v-c_{3} k_{\mathrm{B}}}, \quad \varepsilon_{1}=\frac{c_{2} k_{\mathrm{B}}}{c_{1} v-c_{3} k_{\mathrm{B}}}, \quad \varepsilon_{2}=\frac{c_{1} k_{\mathrm{B}}}{c_{1} v-c_{3} k_{\mathrm{B}}},
$$


thereby implying that the Maxwell equation (3) takes precisely the form (2), or, equivalently, the function $\varphi(x, t ; \eta)$ satisfies the following linear partial differential equation

$$
\eta^{2}\left(c_{1} \frac{\partial^{2} \varphi}{\partial x \partial t}+c_{2} \frac{\partial^{2} \varphi}{\partial x^{2}}\right)+\eta c_{3} \frac{\partial \varphi}{\partial t}+c_{4} \varphi=0 .
$$

Solutions to the above equation with a suitable physical initial condition identify the partition function of the fluid characterised by the internal energy (6). Fundamental properties of the associated thermodynamic system have been studied in [25]. In particular, the case $c_{1} \neq 0, c_{2}=c_{3}=0, c_{4} / c_{1}=a k_{\mathrm{B}}^{-2}$, with $a$ the mean field parameter entering the van der Waals equation of state, has been investigated in [24]. More specifically, based on microscopic arguments, it has been proven that the partition function for the standard van der Waals model satisfies a Klein-Gordon type equation, that is precisely equation (7) with $c_{2}=c_{3}=0$ and $c_{4} / c_{1}=a k_{\mathrm{B}}^{-2}$. In other words, the model (4)-(6) can devise systems whose thermodynamics is anchored to that of van der Waals gases, with novel contributions from effective molecular interactions controlled by the nonvanishing parameters $c_{2}$ and $c_{3}$. Hence, although the condition of linearisability of Maxwell's relation might seem restrictive, this produces a family of models parametrised by three constants, i.e. the ratios $c_{2} / c_{1}, c_{3} / c_{1}, c_{4} / c_{1}$ with $c_{1} \neq 0$, see (6). Moreover, additional functional parameters (specifically, the co-volume) arising form the general solution of the equation (7) or (2) can be fixed via the corresponding initial conditions.

The physical implication of the linearisability request through the nonlinear transformation (5) is twofold. The C-integrability guarantees the existence and explicit evaluation of a Massieu function/thermodynamic potential when finite systems are considered (finite $N$ ). Besides, the typical critical behaviour of fluid systems is reproduced in the thermodynamic limit [3]. Indeed, equation (2) represents a viscous conservation law and as such its generic solution is expected to develop classical shock waves in finite $t$ in the inviscid limit $\eta \rightarrow 0$, corresponding to the thermodynamic limit $N \rightarrow \infty$. That is, in the thermodynamic regime isothermal curves can be interpreted as nonlinear wave solutions to (2) that break in correspondence of the gas-liquid critical point $[21,22]$. Beyond the critical point solutions to the inviscid limit of the equation (2) obtained by setting $\eta=0$ are in fact no longer single-valued. The region where the solution is multi-valued corresponds to the critical region where multiple phases emerge. Such underlying criticality is therefore captured by the implicit solution written in the hodograph form

$$
x+\varepsilon_{0}^{\prime}(v) t=f(v)
$$

that is a solution to the Riemann-Hopf equation

$$
\frac{\partial v}{\partial t}-\varepsilon_{0}^{\prime}(v) \frac{\partial v}{\partial x}=0
$$

obtained from the equation (2) with $\eta=0$, where $f(v)$ is an arbitrary function of volume density and primes denote differentiation with respect to volume, i.e.

$$
\varepsilon_{0}^{\prime}(v)=-\frac{c_{2}}{c_{1}}+\frac{\left(c_{2} c_{3}^{2}+c_{4} c_{1}^{2}\right)}{c_{1}\left(c_{1} v-c_{3} k_{\mathrm{B}}\right)^{2}} k_{\mathrm{B}}^{2} .
$$

Any particular choice of the function $f(v)$ specify the entropic contribution of the ensemble of molecules composing the fluid by means of the relation $f(v)=s^{\prime}(v)$, where $s(v)$ stands for the entropy density. Evaluating the hodograph solution (8) at $t=0$ one has $x=f(\bar{v})$, where $\bar{v}=f^{-1}(x)$ can be interpreted as the volume density of the fluid when temperature and pressure are large but such that the ratio $x=P / T$ is finite. The hodograph function $\Psi(v):=x+\varepsilon_{0}^{\prime}(v) t-f(v)$ thus permits to write down the equation of state for the system under 
investigation as $\Psi(v)=0$. Thermodynamical critical points are associated with the critical sector of the Riemann-Hopf equation (9), defined by solution to the system of simultaneous equations [31]

$$
\Psi(v)=\Psi^{\prime}(v)=\Psi^{\prime \prime}(v)=0 .
$$

In this context, the appearance of coexistence curves is understood as the propagation of weak solutions to (9) in the space of thermodynamic variables [21], the Rankine-Hugoniot shock condition [23] being tantamount to the Maxwell's equal areas rule.

The above framework can be applied to the model of interacting molecules described by an equation of state that, in the high temperature regime, reduces to that of a system of noninteracting hard spheres, i.e. $(\bar{v}-b) x=k_{\mathrm{B}}$, where $b \in \mathbb{R}^{+}$is a parameter proportional to the volume occupied by a molecule of fluid [32]. It then follows that for any value of $t$ the equation of state for the system is given by the hodograph implicit solution $\Psi(v)$ with the particular choice

$$
f^{\mathrm{hs}}(v):=\frac{k_{\mathrm{B}}}{v-b}
$$

and that the system (11) admits the solution given by

$$
\begin{aligned}
x_{c} & =\frac{c_{1}\left[\left(27 b^{2} c_{1}^{2}-54 b c_{1} c_{3} k_{\mathrm{B}}\right) c_{2}+\left(28 c_{2} c_{3}^{2}+c_{1}^{2} c_{4}\right) k_{\mathrm{B}}^{2}\right]}{8 k_{\mathrm{B}}\left(c_{2} c_{3}^{2}+c_{1}^{2} c_{4}\right)\left(b c_{1}-c_{3} k_{\mathrm{B}}\right)}, \\
t_{c} & =\frac{27 c_{1}^{2}\left(b c_{1}-c_{3} k_{\mathrm{B}}\right)}{8 k_{\mathrm{B}}\left(c_{2} c_{3}^{2}+c_{1}^{2} c_{4}\right)}, \\
v_{c} & =3 b-\frac{2 k_{\mathrm{B}} c_{3}}{c_{1}} .
\end{aligned}
$$

The known critical point for a van der Waals fluid is obtained by setting $c_{2}=c_{3}=0$ and $c_{4} / c_{1}=a k_{\mathrm{B}}^{-2}$. All structural constants for the model $c_{j}$ specify the critical points. Domain restrictions arise in the form $c_{3} / c_{1}<b / k_{\mathrm{B}}$ and $c_{2} / c_{1}<0$ upon demanding that $t_{c}>0$ and the partition function be well-behaved [25]. It is also assumed that $c_{2} c_{3}^{2}+c_{1}^{2} c_{4} \neq 0$ as when $c_{2} c_{3}^{2}+c_{4} c_{1}^{2}=0$ the internal energy $\varepsilon_{0}$ depends on volume density linearly and the model does not support phase transitions. In other words the hodograph equation (8) give a travelling wave solution with constant characteristic speed, $x-\frac{c_{2}}{c_{1}} t=f(v)$.

We note that the internal energy density expansion (4) enables one to exploit a formal mathematical analogy between the theory of nonlinear waves and isothermal curves for fluids. The expansion (6) yields to the Maxwell thermodynamic equation (2) that is valid for a global description of a class of fluid systems, inside and outside the critical region. Outside the critical region, the van der Waals equation of state arises at the leading order in the expansion parameter and for the choice of parameters $c_{2}=c_{3}=0$ and $c_{4} / c_{1}=a k_{\mathrm{B}}^{-2}$. Inside the critical region, the analysis of solution through shock-wave techniques provides coexistence lines consistently with Maxwell's equal areas rule.

\section{Symmetry generators of equation (2)}

Symmetry methods are widely applied to examine physical systems. In particular, the adoption of a Lie group-theoretical framework is a well established way to proceed while dwelling upon differential equations. Our purpose here is to characterise the symmetry group of the partial differential equation (2), i.e. the whole class of continuous transformations acting on dependent and independent coordinates that transforms solutions of the equation to other solutions, and to 
infer the possible consequences from a thermodynamic perspective. We point out that analysis is accomplished by assuming that all the parameters $c_{j}$ in equation (2) are non-vanishing and, in addition, $c_{2} c_{3}^{2}+c_{4} c_{1}^{2} \neq 0$.

As discussed at length in a number of works and textbooks, among which we mention [27-29], the task of extracting the symmetry group of a differential equation can be implemented by looking for the admissible symmetry vector fields of the type

$$
W=g_{1}(x, t, v) \frac{\partial}{\partial x}+g_{2}(x, t, v) \frac{\partial}{\partial t}+g_{3}(x, t, v) \frac{\partial}{\partial v} .
$$

Since (2) is a second order equation, to determine the functions $g_{j}(x, t, v)$ that are possibly allowed, the infinitesimal criterion of invariance of the differential equation (2) $\left.\operatorname{pr}^{(2)} W[\Delta]\right|_{\Delta=0}=0$ must be analysed, being $\Delta$ the lhs of (2) and $\operatorname{pr}^{(2)} W$ the so-called second prolongation of the vector field $W$ (see [27]) for details). Since the approach is standard and can be made efficient through symbolic mathematical computations, it is sufficient for the purpose of this paper to report directly the result.

Assuming that none of the coefficients involved in equation (2) do vanish and $c_{2} c_{3}^{2}+c_{4} c_{1}^{2} \neq 0^{4}$ it turns out that the sought symmetry group is generated through the action of the following three vector fields

$$
W_{1}=\frac{\partial}{\partial x}, \quad W_{2}=\frac{\partial}{\partial t}+\frac{c_{2}}{c_{1}} \frac{\partial}{\partial x}, \quad W_{3}=t \frac{\partial}{\partial t}+\left(2 \frac{c_{2}}{c_{1}} t-x\right) \frac{\partial}{\partial x}+\left(v-k_{\mathrm{B}} \frac{c_{3}}{c_{1}}\right) \frac{\partial}{\partial v} .
$$

Each of these operators defines a one-dimensional subgroup of point-transformation, i.e. a group of local transformations $G(\{x, t, v\} ; \lambda)$ whose action on the triplet $\{x, t, v\}$ returns a new triplet of variables that depend on $\{x, t, v\}$ and on a single real parameter. The meaning of operators $W_{1}$ and $W_{2}$ is self-evident because they are associated with rigid translations in the $x$ and $t-\frac{c_{1}}{c_{2}} x$ directions. The corresponding one-dimensional subgroups are settled via $G_{1}\left(\{x, t, v\} ; \lambda_{1}\right)=\left\{x+\lambda_{1}, t, v\right\}$ and $G_{2}\left(\{x, t, v\} ; \lambda_{2}\right)=\left\{x+\frac{c_{2}}{c_{1}} \lambda_{2}, t+\lambda_{2}, v\right\}$. The explicit one-parameter group of symmetry transformation implied by the generator $W_{3}$ is explicitly given as follows:

$G_{3}\left(\{x, t, v\} ; \lambda_{3}\right)=\left\{\mathrm{e}^{-\frac{c_{1}}{c_{2}} \lambda_{3}} x+2 t \frac{c_{2}}{c_{1}} \sinh \left(\frac{c_{1}}{c_{2}} \lambda_{3}\right), \mathrm{e}^{\frac{c_{1}}{c_{2}} \lambda_{3}} t, \mathrm{e}^{\frac{c_{1}}{c_{2}} \lambda_{3}} v+k_{\mathrm{B}} \frac{c_{3}}{c_{1}}\left(1-\mathrm{e}^{\frac{c_{1}}{c_{2}} \lambda_{3}}\right)\right\}$.

A three-parameter group of symmetry thus underlies the differential problem (2), the identity element being recovered by performing the limit where the real parameters $\lambda_{1}, \lambda_{2}$ and $\lambda_{3}$ vanish. It is worth to remark at this stage that the inherent question concerned with the symmetry transformations outcoming whenever a linear combination of symmetry generators (15) is considered finds an immediate answer. Indeed, operators of the form $\tilde{W} \equiv W_{3}+\alpha_{1} W_{1}+\alpha_{2} W_{2}$ with $\alpha_{1,2}$ arbitrary real constants can be actually given a structure of the type $W_{3}$ upon suitable shifts of the real independent variables $x$ and $t$. Of course, the transport of solutions into other

${ }^{4}$ When $c_{2} c_{3}^{2}+c_{4} c_{1}^{2}=0$ infinite symmetries come into play: in addition to three generators of the type (15), the family of symmetry generators

$$
W^{\infty}=\left[G_{1}+\frac{c_{1}}{c_{2}} F_{1}(t)\right] \frac{\partial}{\partial t}+\left[F_{2}\left(-\frac{c_{1}}{c_{2}} x+t\right)+F_{1}(t)\right] \frac{\partial}{\partial x}+\frac{c_{1}}{c_{2}}\left(v-k_{\mathrm{B}} \frac{c_{3}}{c_{1}}\right) F_{2}^{\prime}\left(-\frac{c_{1}}{c_{2}} x+t\right) \frac{\partial}{\partial_{v}}
$$

is found, being $F_{1}, F_{2}$ arbitrary functions of their argument and $G_{1}$ constant. 
solutions through symmetry operations can be ruled by constructing sequences of transformations as well, each of which referring to a given symmetry generator. In such a case, one expects that the order in which different transformations are performed in the sequence matters because while $W_{1}$ and $W_{2}$ are commuting vector fields they both do not commute with $W_{3}$. However, results coming by permutation of the order of transformations in a given sequence are linked each to the other by mere shifts of thermodynamical variables $x$ and $t$. As a consequence, the lesson one ultimately learns from the analysis of equation (2) by means of a group-theoretical approach is that if a function $v=v_{0}(x, t ; \eta)$ solves equation (2) then also

$$
v_{\lambda}(x, t ; \eta)=\mathrm{e}^{\frac{c_{1}}{c_{2}} \lambda_{3}} v_{0}(\tilde{x}, \tilde{t} ; \eta)+\left(1-\mathrm{e}^{\frac{c_{1}}{c_{2}} \lambda_{3}}\right) \frac{c_{3} k_{\mathrm{B}}}{c_{1}}
$$

with

$$
\tilde{x}=\Lambda_{1}+\mathrm{e}^{\frac{c_{1}}{c_{2}} \lambda_{3}} x-2 t \frac{c_{2}}{c_{1}} \sinh \left(\frac{c_{1}}{c_{2}} \lambda_{3}\right), \quad \tilde{t}=\mathrm{e}^{-\frac{c_{1}}{c_{2}} \lambda_{3}} t+\Lambda_{2}
$$

does, being $\left.\left(\Lambda_{1}, \Lambda_{2}, \lambda 3\right), \lambda 3\right)$ a triplet of real deformation parameters. No other local symmetry transformations acting in the space $(x, t, v)$ can be devised when it is assumed that $\eta c_{1} c_{2} c_{3} c_{4} \neq$ 0 along with $c_{2} c_{3}^{2}+c_{4} c_{1}^{2} \neq 0$.

\section{Equations of state and dynamics of critical points under symmetry transformations}

Given a state of the fluid, specified by the variables $(x, t, v)$, the above solutions (17) and (18) parametrised by $\Lambda_{1}, \Lambda_{2}$ and $\lambda_{3}$ describe an orbit of the associate point Lie symmetry group generating a family of equations of state. The action of a symmetry transformation deforms the equations of state as well as the critical properties of the state functions. The approach outlined above in section 3 allows to construct an infinite family of solutions to the Maxwell relation given by the equation (2). Hence, a separate analysis is required to select those solutions that satisfy physical assumptions to possibly capture properties of real fluids.

In the following, we show that in order to ensure that a solution of the group orbit satisfies the required physical properties it is necessary that the seed solutions $v_{0}$ possesses those properties, as for instance suitable asymptotic conditions in the thermodynamical limit. We observe that, regardless of the specific form of the function $v_{0}(x, t ; \eta)$ a symmetry transformation of the form (17) depending on the group parameters $\Lambda_{1}, \Lambda_{2}$ and $\lambda_{3}$ will induce a dependence of critical points on the same parameters.

Let us denote by $\left(x_{c}^{0}, t_{c}^{0}, v_{c}^{0}\right)$ and $\left(x_{c}^{\lambda}, t_{c}^{\lambda}, v_{c}^{\lambda}\right)$ the triplets identifying a critical point for the state functions $v_{0}$ and $v_{\lambda}$ respectively. As the symmetry transformation does not depend on $\eta$ and, therefore, it is not affected by the thermodynamic limit $\eta \rightarrow 0$, the new critical volume is determined by the right-hand side of (17),

$$
v_{c}^{\lambda}=\mathrm{e}^{\frac{c_{1}}{c_{2}} \lambda_{3}} v_{c}^{0}+\left(1-\mathrm{e}^{\frac{c_{1}}{c_{2}} \lambda_{3}}\right) \frac{c_{3} k_{\mathrm{B}}}{c_{1}},
$$

and the transformed critical pressure and temperature are given by

$$
\begin{aligned}
& x_{c}^{\lambda}=\mathrm{e}^{-\frac{c_{1}}{c_{2}} \lambda_{3}}\left(x_{c}^{0}-\Lambda_{1}\right)+2 \frac{c_{2}}{c_{1}} \sinh \left(\frac{c_{1}}{c_{2}} \lambda_{3}\right)\left(t_{c}^{0}-\Lambda_{2}\right), \\
& t_{c}^{\lambda}=\mathrm{e}^{\frac{c_{1}}{c_{2}} \lambda_{3}}\left(t_{c}^{0}-\Lambda_{2}\right),
\end{aligned}
$$


(i.e. equation (18) with the replacements $\tilde{x} \rightarrow x_{c}^{0}, \tilde{t} \rightarrow t_{c}^{0}, x \rightarrow x_{c}^{\lambda}, t \rightarrow t_{c}^{\lambda}$ ). It is worth noting that the symmetry approach allows to study a family of models and their critical properties via the initial condition on the equation (2).

It is also interesting to study the effect of the group transformation at the level of the partition function and Gibbs free energy potential. This step is in fact propaedeutic to the determination of the phase-diagrams for the deformed equations of state $v_{\lambda}$. As by definition we have

$$
v_{\lambda}(x, t ; \eta)=-\eta k_{\mathrm{B}} \frac{\partial}{\partial x} \log \varphi_{\lambda}(x, t ; \eta)
$$

where $\varphi_{\lambda}(x, t ; \eta)$ denotes the solution to equation $(7)$, the partition function $\varphi_{\lambda}(x, t ; \eta)$ is given, up to a constant factor, by ${ }^{5}$

$$
\varphi_{\lambda}(x, t ; \eta)=\int_{b_{\lambda}}^{\infty} \mathrm{d} v \exp \left(-\frac{\Omega_{\lambda}}{\eta k_{\mathrm{B}}}\right),
$$

where $\Omega_{\lambda}$ plays the role of the Gibbs free energy density of the form

$$
\Omega_{\lambda}=x v+\varepsilon_{0}(v) t-s_{\lambda}(v) .
$$

The deformed entropy density obtained from $s_{0}(v)$ and associated with function $v_{0}(x, t)$ is given by

$$
\begin{aligned}
s_{\lambda}(v)= & s_{0}\left(\mathrm{e}^{-\frac{c_{1}}{c_{2}} \lambda_{3}}\left[v-\frac{c_{3}}{c_{1}} k_{\mathrm{B}}\left(1-\mathrm{e}^{\frac{c_{1}}{c_{2}} \lambda_{3}}\right)\right]\right)+\mathrm{e}^{-\frac{c_{1}}{c_{2}} \lambda_{3}}\left(\frac{c_{2}}{c_{1}} \Lambda_{2}-\Lambda_{1}\right) v \\
& +\frac{\left(c_{2} c_{3} v+c_{1} c_{4} k_{\mathrm{B}}\right)}{c_{1}\left(c_{1} v-c_{3} k_{\mathrm{B}}\right)} \mathrm{e}^{\frac{c_{1}}{c_{2}} \lambda_{3}} \Lambda_{2} k_{\mathrm{B}} .
\end{aligned}
$$

The quantity $b_{\lambda}=\mathrm{e}^{\frac{c_{1}}{c_{2}} \lambda_{3}} b+\frac{k_{\mathrm{B}} c_{3}}{c_{1}}\left(1-\mathrm{e}^{\frac{c_{1}}{c_{2}} \lambda_{3}}\right)$ corresponds to the minimum value of the deformed volume density. The domain restrictions $\frac{c_{2}}{c_{1}}<0$ and $\frac{c_{3}}{c_{1}}<\frac{b}{k_{\mathrm{B}}}$ on the structural constants $c_{j}$ 's ensure that $b_{\lambda}$ is positive, being lower than the minimum value for the seed solution $v_{0}$ for positive values of $\lambda_{3}$.

The formula (20) represents a smooth map providing a family of deformed partition functions starting from a seed partition function. The asymptotic evaluation of deformed partition functions (20) is then obtained by the standard Laplace formula, and implies the equation

$$
\Omega_{\lambda}^{\prime}=x+\varepsilon_{0}^{\prime}(v) t-s_{\lambda}^{\prime}(v)=0,
$$

where $\Omega_{\lambda}$ is given by (21) with the entropic term (22) and $\Omega_{\lambda}^{\prime}=\frac{\partial \Omega_{\lambda}}{\partial v}$. Equation (23) gives the possible equations of state along the symmetry group orbits, with the function $f_{\lambda}(v) \equiv s_{\lambda}^{\prime}(v)$ assigning the entropic contribution to the equation of state. Remarkably, the derivative of the potential $\Omega_{\lambda}$ with respect to the volume coincides with the hodograph function introduced in section 2 .

${ }^{5}$ If $\varphi_{0}(x, t ; \eta)$ solves equation (7) then

$$
\varphi_{\lambda}(x, t ; \eta)=\varphi_{0}(\tilde{x}, \tilde{t} ; \eta) \exp \left\{\frac{c_{3}}{\eta c_{1}}\left(1-\mathrm{e}^{-\frac{c_{1}}{c_{2}} \lambda_{3}}\right) \tilde{x}+2 \frac{c_{2} c_{3}}{c_{1}^{2} \eta}\left[\cosh \left(\frac{c_{1}}{c_{2}} \lambda_{3}\right)-1\right] \tilde{t}\right\}
$$

where $\varphi_{0}(\tilde{x}, \tilde{t} ; \eta)$ stands for the partition function of original model with arguments $x, t$ replaced by the functions $\tilde{x}=$ $\tilde{x}(x, t)$ and $\tilde{t}=\tilde{t}(x, t)$ given in (18). By taking $\varphi_{0}(x, t ; \eta)=\int_{b}^{\infty} \mathrm{d} v \exp \left(-\frac{\Omega_{0}}{\eta k_{\mathrm{B}}}\right)$ with $\Omega_{0}=x v+\varepsilon_{0}(v) t-s_{0}(v)$ as in [25], one can write $\varphi_{\lambda}(x, t ; \eta)$ in the form (20) and (21). 

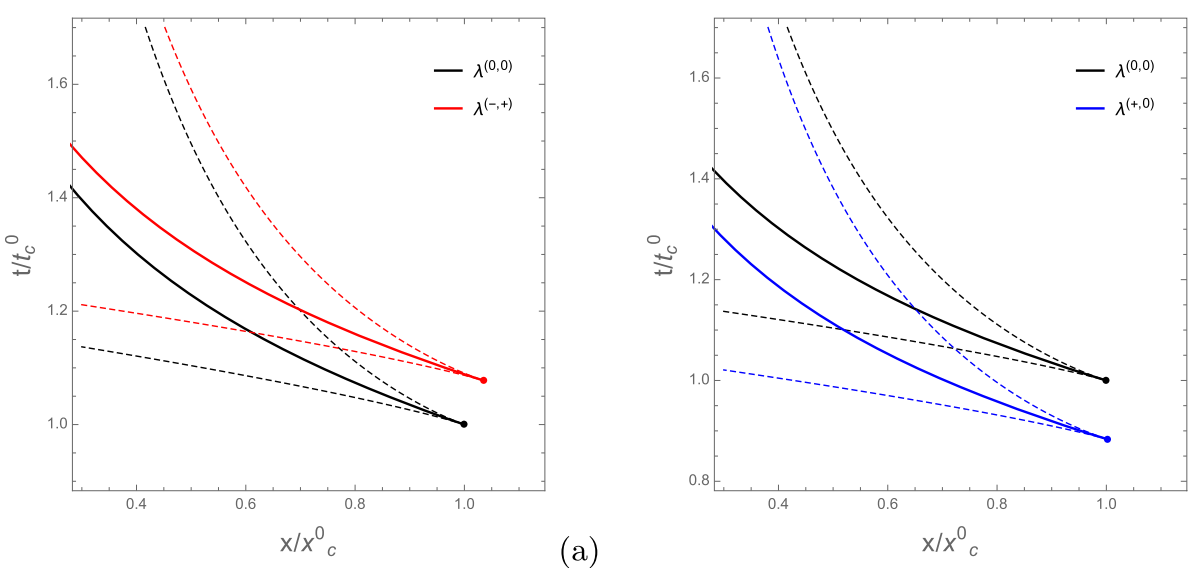

(b)

Figure 1. Examples of dynamics of shock trajectory under symmetry group action. The coexistence line (solid line) originates from the critical point (solid circle) and crosses the critical region (delimited by the dashed lines). Black: seed solution to (2) $v_{0}$ that captures the critical point of the Soave-Redlich-Kwong equation of state for hydrogen gas $\left(b=4.419 \times 10^{-29} \mathrm{~m}^{3}, c_{2} / c_{1}=-8452 \mathrm{~Pa}, c_{3} / c_{1}=-1.356 \times 10^{-6} \mathrm{~K} \mathrm{~Pa}^{-1}\right.$ and $\left.c_{4} / c_{1}=a k_{\mathrm{B}}^{-2}=3.582 \times 10^{-4} \mathrm{~K}^{2} \mathrm{~Pa}^{-1}\right)$. Red: new solution $v_{\lambda}$ gained for $\Lambda_{1}=\frac{c_{2}}{c_{1}} \Lambda_{2}$ and $\lambda^{(-,+)}=\left(\Lambda_{2}=-0.005 \mathrm{~K}^{-1}, \lambda_{3}=300 \mathrm{~Pa}\right)$. Blue: $v_{\lambda}$ when $\Lambda_{1}=\frac{c_{2}}{c_{1}} \Lambda_{2}$ and $\lambda^{(+, 0)}=$ $\left(\Lambda_{2}=0.005 \mathrm{~K}^{-1}, \lambda_{3}=0\right)$. For the sake of homogeneous schematics, thereinafter legend $\lambda^{(0,0)}$ is used for the $v_{0}$ 's to stress that the case is concerned with no deformation, $\Lambda_{1}=\Lambda_{2}=\lambda_{3}=0$.

We note that the expressions (19) require that the liquid-to-gas critical point occurs in the physical domain $x, t \geqslant 0$ when the deformation parameters satisfy the following constraints

$$
\Lambda_{2} \leqslant t_{c}^{0}, \quad \Lambda_{1} \leqslant x_{c}^{0}-\frac{c_{2}}{c_{1}}\left(1-\mathrm{e}^{\frac{2 c_{1}}{c_{2}} \lambda_{3}}\right)\left(t_{c}^{0}-\Lambda_{2}\right)
$$

A further physical constraint corresponds to the request that in the limit of zero pressure the infinite volume density is implied. If the starting entropy $s_{0}$ shapes an equation of state that meets the above physical specifications, the function $f(v)=s_{0}^{\prime}(v)$ goes to zero in the limit $v \rightarrow \infty$, the pressure accordingly vanishing as $t \rightarrow 0$. Under these circumstances, by fixing $c_{1} \Lambda_{1}=c_{2} \Lambda_{2}$ we retrieve the desired asymptotics either for the new equation of state.

Let us consider, for example, the case of the van der Waals equation of state as a seed solution in the limit $\eta \rightarrow 0$ for which the entropy $s_{0}$ is given by hard sphere formula $s^{\text {hs }}(v)=k_{\mathrm{B}} \log (v-$ $b$ ). Hence, the corresponding deformation of the function $f^{\mathrm{hs}}(v)=k_{\mathrm{B}}(v-b)^{-1}$ (equation (12)) is given by

$$
f_{\lambda}^{\mathrm{hs}}(v)=\frac{k_{\mathrm{B}}}{v-b_{\lambda}}+\mathrm{e}^{-\frac{c_{1}}{c_{2}} \lambda_{3}}\left(\frac{c_{2}}{c_{1}} \Lambda_{2}-\Lambda_{1}\right)-\frac{k_{\mathrm{B}}^{2}\left(c_{1}^{2} c_{4}+c_{2} c_{3}^{2}\right)}{c_{1}\left(c_{1} v-c_{3} k_{\mathrm{B}}\right)^{2}} \mathrm{e}^{\frac{c_{1}}{c_{2}} \lambda_{3}} \Lambda_{2} .
$$

Evaluating the deformed equation of state $x+\varepsilon_{0}^{\prime}(v) t=f_{\lambda}^{\text {hs }}(v)$ as $t \rightarrow 0$ and $v \rightarrow \infty$ we have

$$
x_{\infty}=\lim _{v \rightarrow \infty} f_{\lambda}^{\mathrm{hs}}(v)=\mathrm{e}^{-\frac{c_{1} \lambda_{3}}{c_{2}}}\left(\frac{c_{2}}{c_{1}} \Lambda_{2}-\Lambda_{1}\right) .
$$



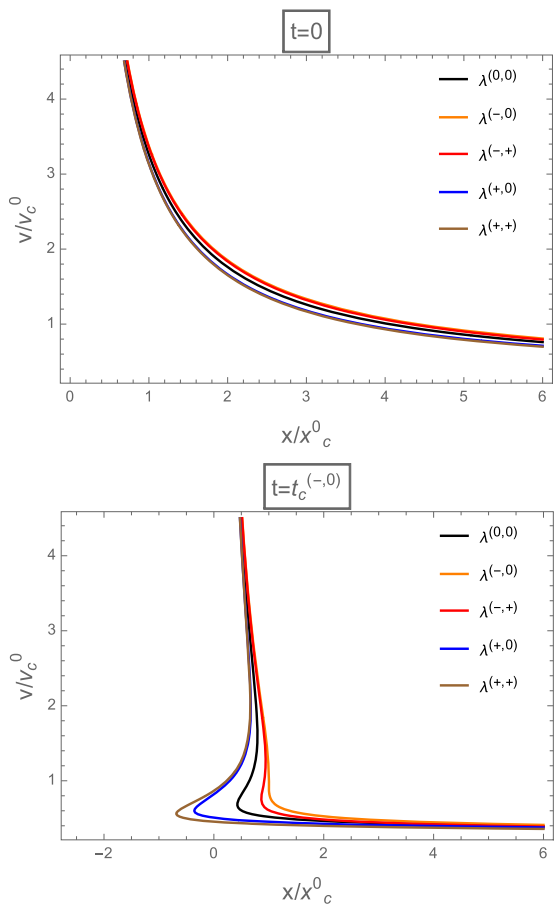

(a)
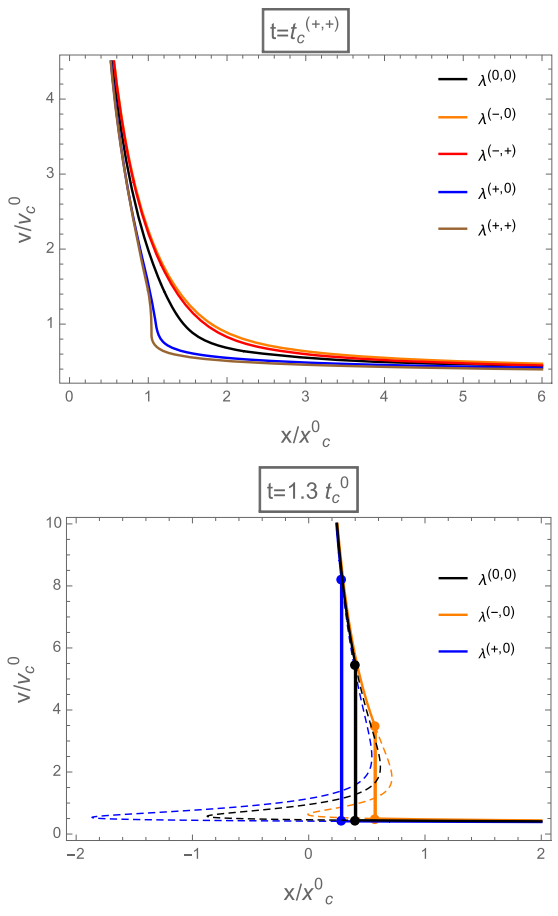

(b)

(c)

Figure 2. Dynamics of isothermal curves for the solution $v_{0}$ (black) that captures the critical point of the Soave-Redlich-Kwong equation of state for hydrogen gas and new equations of state $v_{\lambda}$ (brown/blue/red/orange) obtained through nontrivial pairs $\left(\Lambda_{2}, \lambda_{3}\right)$ jointly with the ansatz $\Lambda_{1}=\Lambda_{2} c_{2} / c_{1}$. The plots refer to the pairs of deformation parameters $\lambda^{(+,+)}=\left(0.005 \mathrm{~K}^{-1}, 300 \mathrm{~Pa}\right)$ (brown), $\lambda^{(+, 0)}=\left(0.005 \mathrm{~K}^{-1}, 0 \mathrm{~Pa}\right)$ (blue), $\lambda^{(-,+)}=\left(-0.005 \mathrm{~K}^{-1}, 300 \mathrm{~Pa}\right)\left(\right.$ red) and $\lambda^{(-, 0)}=\left(-0.005 \mathrm{~K}^{-1}, 0 \mathrm{~Pa}\right)$ (orange). (a) Isothermal curves at $t=0$, corresponding to infinite temperature. (b) Isothermal curves at $t_{c}^{(+,+)} \approx 0.85274 t_{c}^{0}$, i.e. at the critical temperature of solution $v_{\lambda}$ obtained with parameters $\lambda^{(+,+)}$. (c) Isothermal curves at the critical temperature of equation of state $v_{\lambda}$ with the choice $\lambda^{(-, 0)}, t_{c}^{(-, 0)} \approx 1.11645 t_{c}^{0}$. (d) Physical isothermal curves all beyond their corresponding critical temperature: solid vertical lines are located in correspondence of the shock positions and allow to remove the multi-valuedness of the solution by replacing its oscillating middle part (dashed curves) with a finite jump in the volume density.

The condition that the pressure must vanish when the volume diverges implies the constraint $c_{2} \Lambda_{2}=c_{1} \Lambda_{1}$. In the limit of infinite pressure the volume density attains its minimum value $b_{\lambda}$, for any choice of acceptable parameters.

We now study the phase diagrams associated to the deformed equations of state. Figure 1 shows the coexistence line corresponding to the shock trajectory and the critical region enclosed by the general fold [33] of the equation, i.e. the solutions of the system $\Psi_{\lambda}(v)=$ $\Psi_{\lambda}^{\prime}(v)=0$ for the hodograph function $\Psi_{\lambda}(v):=x+\varepsilon_{0}^{\prime}(v) t-f_{\lambda}^{\text {hs }}(v)$. The chosen numerical values for the structural parameters are such that the seed solution $v_{0}$ to equation (2) provides the critical point of the Soave-Redlich-Kwong equation of state. The figure illustrates the effect of the deformation of this solution induced by the symmetry transformation (17) and (18) as the parameters $\Lambda_{2}$ and $\lambda_{3}$ vary such that the constraint $c_{1} \Lambda_{1}=c_{2} \Lambda_{2}$ is fulfiled in terms of the resulting displacement of the critical points and the coexistence curve in the plane 
of thermodynamical variables $(x, t)$. The coexistence curve gives the trajectory of the shock emerging from the critical point as specified by the asymptotic evaluation of the integral (20) for $t>t_{c}$. More specifically, for small $\eta$ we have

$$
\varphi_{\lambda}(x, t ; \eta) \simeq \sum_{k} \sqrt{\frac{2 \pi \eta k_{\mathrm{B}}}{\Omega_{\lambda, k}^{\prime \prime}}} \mathrm{e}^{-\frac{\Omega_{\lambda, k}}{\eta k_{\mathrm{B}}}},
$$

where the sum index $k$ runs over the local minima at fixed pressure and temperature of the Gibbs free energy (21). Along the coexistence lines we have for all pairs of solutions associated to gas and the liquid phases

$$
\Omega_{\lambda, j}=\Omega_{\lambda, k}
$$

The effect of deformations on the isothermal curves is illustrated in figure 2 where we chose four different non-trivial sets of deformation parameters $\left(\Lambda_{1}, \Lambda_{2}, \lambda_{3}\right)$. Figure 2(a) shows that in the limit of infinite temperature $t \rightarrow 0$, isotherms associated to the equation of state for $v_{\lambda}$ 's depart from the ones associated to $v_{0}$ but overall preserve their qualitative features. As the temperature decreases isothermal curves develop a multivalued behaviour as functions of $x$. However, the curves differ quantitatively as each solutions evolves at a different characteristic speed, which explains the shift in the position of the shock profile. In particular, for fixed symmetry parameters, there is a temperature interval where the two solutions $v_{0}$ and $v_{\lambda}$ exhibit opposed behaviours. Indeed, as illustrated in figure 2(b), the volume density $v_{\lambda}$ associated with the pair $\left(\Lambda_{2}, \lambda_{3}\right)$ from set $\lambda^{(+,+)}$develops a gradient catastrophe at $t=t_{c}^{(+,+)}$, while the seed solution and the solutions corresponding to the choice of parameters $\lambda^{(+, 0)}, \lambda^{(-,+)}$and $\lambda^{(-, 0)}$ remain single-valued. For temperatures below the critical value, the multivalued volume density profile $v_{\lambda}$ with $\lambda^{(+,+)}$is replaced by classical shock. At $t=t_{c}^{0}$, it is the seed solution $v_{0}$ that experiences a gradient catastrophe, while, for example, the solutions $v_{\lambda}$ constructed with negative $\Lambda_{2}$ are single-valued. At sufficiently low temperatures single-valuedness is lost for all the five solutions (see figure 2(c)), and physical isotherms are given by shock waves travelling towards lower pressures. Remarkably, the location of the shocks fulfils Maxwell's equal areas rule. An illustrative example of the application of the shock fitting procedure to determine coexistence lines at fixed temperature is explicitly demonstrated in figure 2(d). In this respect, it is important to note how solutions $v_{\lambda}$ differ at low temperature. In this regime the contribution from the internal energy is dominant over the entropic one. However, evidently different realisations of the phase transition result from minor differences for the entropic term $s_{\lambda}^{\prime}(v)$, which is the initial datum for the nonlinear differential equation governing the equation of state and establishes the fluid's behaviour far from the critical region.

\section{Conclusions}

We have studied the Lie-point symmetries generated by infinitesimal operators giving finite group transformations for a thermodynamic model, recently introduced in [25], based on the differential equation (2) for the volume density. The model provides the first derivation of a new extension of the van der Waals model as studied in [21] valid in the critical region and it is proven to be effective in the construction of a new interpolating model compatible with empirical models, such as the Peng-Robinson and the Soave-Redlich-Kwong. We found that a rather restricted set of point symmetries generators underlies the differential problem (2) with all four nonvanishing structural constants $c_{j}$, and such that $c_{2} c_{3}^{2}+c_{4} c_{1}^{2} \neq 0$ (a necessary condition for the generation of critical points). The class of point symmetries obtained underpin invariance 
under translations and scalings, and a linear mixing of the independent thermodynamical variables $x=P / T$ and $t=1 / T$. The equation (17) gives a solution depending on three real parameters providing continuous deformations of solutions to the differential equation (2). Tuning the symmetry parameters permits to interpolate between existing models for real fluid matching qualitative properties and critical points. Critical points obtained from deformed equations of state depend on the parameters realising the action of the symmetry group and are connected to the critical points of the seed solution $v_{0}(x, t)$ to which (17) is applied, equation (19). The deformations induced by the action of the symmetry group allow to model significant deviations from the seed solution in the vicinity of the critical point consistently with the behaviour of the van der Waals model at high temperature.

The problem of constructing suitable partial differential equations for state functions of thermodynamic systems and the study of critical properties in terms of critical asymptotics of the solutions to these equations is an active field of research which brought further insights on a variety of classical systems, see e.g. [34-39], and appears to be promising for the study of complex systems [40,41]. Studies exploiting the Lie symmetry analysis can be therefore carried out for other systems of physical interest. Natural developments include the study of composite systems, such as fluid mixtures [42], and nematic fluids [43].

\section{Acknowledgments}

FG and AM acknowledge the hospitality of the Lecce's division of I.N.F.N. and of the Department of Mathematics and Physics 'Ennio De Giorgi' of the University of Salento. GL acknowledges the hospitality of the Department of Mathematics, Physics and Electrical Engineering of Northumbria University. GL and LM are partially supported by INFN IS-MMNLP. AM is supported by the Leverhulme Trust Research Project Grant RPG 2017-228. AM is also grateful to the London Mathematical Society, the Royal Society International Exchanges Grant IESR2-170116, GNFM — Gruppo Nazionale per la Fisica Matematica, INdAM (Istituto Nazionale di Alta Matematica) for supporting activities that contributed to the research reported in this paper.

\section{Data availability statement}

All data that support the findings of this study are included within the article (and any supplementary files).

\section{ORCID iDs}

Francesco Giglio (D) https://orcid.org/0000-0003-1131-1560

Giulio Landolfi (D) https://orcid.org/0000-0001-6699-7876

Luigi Martina (1) https://orcid.org/0000-0002-2858-7320

Antonio Moro (i) https://orcid.org/0000-0002-4901-8573

\section{References}

[1] Lopez-Echeverry J S, Reif-Acherman S and Araujo-Lopez E 2017 Peng-Robinson equation of state: 40 years through cubics Fluid Phase Equilib. 447 39-71

[2] Levelt Sengers J M H 2003 How Fluids Unmix: Discoveries by the School of van der Waals and Kamerlingh Onnes (Amsterdam: Edita KNAW) 
[3] Hansen J P and McDonald I R 2013 Theory of Simple Liquids (London: Academic)

[4] Rowlinson J S and van der Waals J D 1988 On the Continuity of the Gaseous and Liquid States (Holland: Elsevier)

[5] Johnston D C 2014 Advances in Thermodynamics of the van der Waals Fluid (San Rafael: Morgan and Claypool Publishers)

[6] Soave G 1972 Equilibrium constants from a modified Redlich-Kwong equation of state Chem. Eng. Sci. 27 1197-203

[7] Peng D-Y and Robinson D B 1976 A new two-constant equation of state Ind. Eng. Chem. Fundam. 15 59-64

[8] Martin J J 1979 Cubic equations of state-which? Ind. Eng. Chem. Fundam. 18 81-97

[9] Kubic W L 1982 A modification of the Martin equation of state for calculating vapour-liquid equilibria Fluid Phase Equilib. 9 79-97

[10] Forero G L A and Velásquez J J A 2013 A modified Patel-Teja cubic equation of state: I. Generalized model for gases and hydrocarbons Fluid Phase Equilib. 342 8-22

Forero G L A and Velásquez J J A 2014 A modified Patel-Teja cubic equation of state: II. Parameters for polar substances and its mixtures Fluid Phase Equilib. 364 75-87

[11] Zhong W, Xiao C and Zhu Y 2017 Modified van der Waals equation and law of corresponding states Physica A 471 295-300

[12] Yan W, Varzandeh F and Stenby E H 2015 PVT modeling of reservoir fluids using PC-SAFT EoS and Soave-BWR EoS Fluid Phase Equilib. 386 96-124

[13] Estela-Uribe J F 2017 Fundamental multiparameter and association equation of state for ethanol Fluid Phase Equilib. 452 74-93

[14] Farzi N and Hosseini P 2016 A new equation of state for gaseous, liquid, and supercritical fluids Fluid Phase Equilib. 409 59-71

[15] Schmid B and Gmehling J 2019 From van der Waals to VTPR: the systematic improvement of the van der Waals equation of state J. Supercrit. Fluids 55 438-47

[16] Percus J K and Yevick G J 1958 Analysis of classical statistical mechanics by means of collective coordinates Phys. Rev. 110 1-13

[17] Hayter J B and Penfold J 1981 An analytic structure factor for macroion solutions Mol. Phys. 42 109-18

[18] Mangold F, Pilz S, Bjelić S and Vogel F 2019 Equation of state and thermodynamic properties for mixtures of $\mathrm{H}_{2} \mathrm{O}, \mathrm{O}_{2}, \mathrm{~N}_{2}$, and $\mathrm{CO}_{2}$ from ambient up to $1000 \mathrm{~K}$ and $280 \mathrm{MPa} J$. Supercrit. Fluids 153104476

[19] Nezbeda I, Melnyk R and Trokhymchuk A 2010 A new concept for augmented van der Waals equations of state J. Supercrit. Fluids $\mathbf{5 5} 448-54$

[20] Wang C-Y and Chen Y-Y 2018 A mechanical approach to one-dimensional interacting gas Chin. J. Phys. 56 2006-21

[21] Moro A 2014 Shock dynamics of phase diagrams Ann. Phys. 343 49-60

[22] De Nittis G and Moro A 2012 Thermodynamic phase transitions and shock singularities Proc. R. Soc. A $468701-19$

[23] Whitham G B 1974 Linear and Nonlinear Waves (New York: Wiley)

[24] Barra A and Moro A 2015 Exact solution of the van der Waals model in the critical region Ann. Phys. 359 290-9

[25] Giglio F, Landolfi G and Moro A 2016 Integrable extended van der Waals model Physica D 333 293-300

[26] Gordin V 2000 Mathematical Problems and Methods of Hydrodynamic Weather Forecasting (London: Gordon and Breach)

[27] Olver P 1993 Applications of Lie Groups to Differential Equations (Graduate Texts in Mathematics) vol 107 (New York: Springer)

[28] Stephani H 1989 Differential Equations: Their Solution Using Symmetries (Cambridge: Cambridge University Press)

[29] Ovsiannikov L V 1982 Group Analysis of Differential Equations (New York: Academic)

[30] Calogero F 1991 Why are certain nonlinear PDEs both widely applicable and integrable? What Is Integrability? ed V E Zakharov (Berlin: Springer) pp 1-62

[31] Kodama Y and Konopelchenko B G 2002 Singular sector of the Burgers-Hopf hierarchy and deformations of hyperelliptic curves J. Phys. A: Math. Gen. 35 L489

[32] Callen H B 1985 Thermodynamics and an Introduction to Thermostatistics (New York: Wiley)

[33] Arnol'd V I 1983 Catastrophe Theory (Berlin: Springer) 
[34] Lorenzoni P and Moro A 2019 An exact study of phase transitions in mean field Potts models Phys. Rev. E 100022103

[35] De Matteis G, Giglio F and Moro A 2018 Exact equations of state for nematics Ann. Phys., NY 396 386-96

[36] Choquard P and Wagner J 2004 On the mean field interpretation of Burgers equation J. Stat. Phys. $116843-53$

[37] Barra A, Del Ferraro G and Tantari D 2013 Mean field spin glasses treated with PDE techniques Eur. Phys. J. B 86332

[38] Agliari E, Barra A, Landolfi G, Murciano S and Perrone S 2018 Complex reaction kinetics in chemistry: a unified picture suggested by mechanics in physics Complexity 20187423297

[39] Biondini G, Moro A, Prinari B and Sankevich O 2021 p-star models, mean field random networks and the heat hierarchy (arXiv:2105.09479)

[40] Benassi C and Moro A 2020 Thermodynamic limit and dispersive regularisation in matrix models Phys. Rev. E 101052118

[41] Benassi C, Dell'Atti M and Moro A 2021 Symmetric matrix ensemble and integrable hydrodynamic chains (arXiv:2101:10232)

[42] Levelt Sengers J M H, Kayser R F, Peters C J and White H J 2000 Equations of State for Fluids and Fluid Mixtures (Amsterdam: Elsevier)

[43] De Gennes P G and Prost J 1995 The Physics of Liquid Crystals vol 83 (Oxford: Oxford University Press) 